\title{
WEDDERBURN DECOMPOSITIONS OF COMMUTATIVE BANACH ALGEBRAS
}

\author{
MICHEL SOLOVEJ \\ (Communicated by Palle E. T. Jorgensen)
}

\begin{abstract}
We prove that if $A$ is a commutative Banach algebra with $\operatorname{rad}(A)^{2}$ $=0$ and $A / \operatorname{rad}(A)=C([0,1])$ for the unit interval $[0,1]$, then $A$ has a strong Wedderburn decomposition.
\end{abstract}

\section{INTRODUCTION}

A Banach algebra $A$ is said to have a Wedderburn decomposition (WD) if there is a subalgebra $B \subseteq A$ such that $A$ is the semidirect product of $B$ and the Jacobson $\operatorname{radical} \operatorname{rad}(A)$, i.e.,

$$
A=B \oplus \operatorname{rad}(A),
$$

with multiplication in $A$ given by $\left(b_{1}+r_{1}\right)\left(b_{2}+r_{2}\right)=b_{1} b_{2}+\left(b_{1} r_{2}+r_{1} b_{2}+r_{1} r_{2}\right)$. If the subalgebra $B$ is closed, then $A$ is said to have a strong Wedderburn decomposition (SWD).

The Wedderburn principal theorem states that finite-dimensional (complex) algebras have Wedderburn decomposition. For infinite-dimensional Banach algebras the situation is not so simple. Examples are given of Banach algebras without Wedderburn decomposition (e.g., [3]) and also of Banach algebras having (WD) but not (SWD) (e.g., [6]).

This paper is concerned with the existence of Wedderburn decomposition in unital commutative Banach algebras $A$ such that $A / \operatorname{rad}(A)=C\left(\Phi_{A}\right)$ where $\Phi_{A}$ is the maximal ideal space of $A$. The research into this area was initiated by Bade and Curtis ([1]) who among other things showed that if $A$ is of this type and furthermore satisfies that $\Phi_{A}$ is totally disconnected and has nilpotent radical, then $A$ has (SWD). In [1] Bade and Curtis gave an example of a commutative Banach algebra $\mathscr{U}$ such that $\mathscr{U} / \operatorname{rad}(\mathscr{U})=C(\Omega)$ for a totally disconnected compact space $\Omega$ but such that $\mathscr{U}$ does not have a Wedderburn decomposition. Hence the condition on nilpotency of the radical is not redundant. It follows from [9, Theorem 3.2] that for every infinite compact Hausdorff space $K$, there exists a Banach algebra $A$ such that $\operatorname{rad}(A)^{2}=0$ and

Received by the editors September 10, 1993.

1991 Mathematics Subject Classification. Primary 46J40; Secondary 46J10.

Supported by the Danish Natural Science Research Council and the Danish Research Academy. 
$A / \operatorname{rad}(A)=C(K)$ but such that $A$ does not have a strong Wedderburn decomposition. In general, however, $A$ is not commutative, and so commutativity is essential for the Bade-Curtis result.

For a Banach algebra $A$ let $\mathscr{I}(A)$ denote the set of idempotents in $A$. The result of Bade and Curtis is proved by showing that the closed linear span of $\mathscr{I}(A)$ is a closed subalgebra complementary to the radical. Hence the existence of many idempotents in the algebra plays an important role. Even so it is at the present time not known whether the assumption that the space $K$ is totally disconnected is needed. We pose this as an open problem.

Question 1.1. Let $A$ be a commutative unital Banach algebra with $\operatorname{rad}(A)^{n}=0$ and such that the Gelfand transform $\mathscr{G}: A \rightarrow C\left(\Phi_{A}\right)$ is onto. Does $A$ necessarily have a strong Wedderburn decomposition?

The problem has not been explicitly posed before, but it has been implicitly in the literature for quite a long time (see, e.g., [7, p. 410]). We concentrate on the case $n=2$. This is not a serious restriction, because of the following lemma which is essentially due to Hochschild ([8]). We give the proof for the sake of completeness.

Lemma 1.2. Let $B$ be a semisimple Banach algebra and suppose that all Banach algebras $A$ satisfying that $A / \operatorname{rad}(A)$ is isomorphic to $B$ and $\operatorname{rad}(A)^{2}=0$ have $(S W D)$. Then all Banach algebras $A$ such that $A / \operatorname{rad}(A)$ is isomorphic to $B$ and such that $\operatorname{rad}(A)^{n}=0$ for some $n \in \mathbb{N}$ will have $(S W D)$.

Proof. We prove this by induction on $n \in \mathbb{N}$. For $n=1$ and $n=2$ there is nothing to prove. Let $n>2$ and suppose that the lemma has been proved for all integers less than $n$.

Set $\mathfrak{A}=A /\left(\overline{\operatorname{rad}(A)^{2}}\right)$ and let $\pi: A \rightarrow \mathfrak{A}$ be the quotient map. Then clearly $\mathfrak{A}$ is a Banach algebra such that $\operatorname{rad}(\mathfrak{A})^{2}=0$ and $\mathfrak{A} / \operatorname{rad}(\mathfrak{A})$ is isomorphic to $B$. Consequently there exists a closed subalgebra $\mathfrak{A}_{0}$ of $\mathfrak{A}$ such that $\mathfrak{A}=$ $\mathfrak{A}_{0} \oplus \operatorname{rad}(\mathfrak{A})$.

Set $A_{0}=\pi^{-1}\left(\mathfrak{A}_{0}\right)$. Then

$$
\overline{\operatorname{rad}(A)^{2}} \subseteq A_{0} \cap \operatorname{rad}(A) \subseteq \operatorname{rad}\left(A_{0}\right) \subseteq \overline{\operatorname{rad}(A)^{2}} .
$$

Hence $A_{0}$ is a Banach algebra such that $\operatorname{rad}\left(A_{0}\right)^{n-1}=0$ and such that $A_{0} / \operatorname{rad}\left(A_{0}\right)$ is isomorphic to $B$. By the inductive hypothesis there is a closed subalgebra $A^{\prime}$ of $A_{0}$ such that $A_{0}=A^{\prime} \oplus \operatorname{rad}\left(A_{0}\right)$. It follows easily that $A=A^{\prime} \oplus \operatorname{rad}(A)$ is a (SWD).

In [11] the present author showed that for every infinite compact Hausdorff space $K$ there exist unital commutative algebras $\mathscr{A}$ such that $\mathscr{A} / \operatorname{rad}(\mathscr{A})=$ $C(K)$ and $\operatorname{rad}(\mathscr{A})^{2}=0$ but such that $\mathscr{A}$ does not have (WD). However, these algebras cannot be normed as Banach algebras ([11]).

For the rest of this paper $K$ is a compact Hausdorff space and $A$ is a unital commutative Banach algebra with radical $R$ such that $A / R$ is isometrically isomorphic to $\left(C(K),\|\cdot\|_{\infty}\right)$ and $R^{2}=0$. Throughout $\pi$ will denote the quotient map $\pi: A \rightarrow A / R$.

In Section 6 we shall restrict ourselves to the case where $K$ is the unit interval $[0,1]$. The main result is that in this case $A$ has a strong Wedderburn decomposition. Note that $A$ has only one non-zero idempotent. 


\section{THE SECOND DUAL}

Consider the second dual $A^{* *}$ as a Banach algebra with the first Arens product (see [4, p. 50]). We shall see that $A^{* *}$ is a Banach algebra with many idempotents. In fact we have that $A^{* *} / R^{\perp \perp}$ is isometrically isomorphic to $C(\Omega)$ for some extremely disconnected compact Hausdorff space $\Omega$. Furthermore we have that $R^{\perp \perp}=\operatorname{ker}\left(\pi^{* *}\right)$, and hence $R^{\perp \perp}$ is a closed ideal in $A^{* *}$. From the fact that $A^{* *} / R^{\perp \perp}=C(\Omega)$ is semisimple it easily follows that $\operatorname{rad}\left(A^{* *}\right) \subseteq R^{\perp \perp}$. We shall now show that in the case where $R^{2}=0$ then actually $\operatorname{rad}\left(A^{* *}\right)=R^{\perp \perp}$.

Lemma 2.1. Let $A$ be a Banach algebra and let $B \subseteq A$ be a closed subalgebra such that $B^{n}=0$ for some $n \in \mathbb{N}$. Then $B^{\perp \perp} \subseteq A^{* *}$ satisfies $\left(B^{\perp \perp}\right)^{n}=0$.

Proof. By the Hahn-Banach theorem we have weak ${ }^{*}-\operatorname{cl}(B)=B^{\perp \perp}$.

Let $b_{1}, \ldots, b_{n} \in B^{\perp \perp}$, and choose nets $\left\{b_{\alpha_{1}}\right\}, \ldots,\left\{b_{\alpha_{n}}\right\}$ in $B$ such that $b_{\alpha_{i}} \rightarrow b_{i}$ weak $^{*}(i=1, \ldots, n)$. Then we have

$$
b_{1} \cdots b_{n}=\lim _{\alpha_{1}} \lim _{\alpha_{2}} \ldots \lim _{\alpha_{n}} b_{\alpha_{1}} \cdots b_{\alpha_{n}}=0 \text {. }
$$

Note that $R^{\perp \perp}$ is an ideal in $A^{* *}$ with $\left(R^{\perp \perp}\right)^{2}=0$ and so $R^{\perp \perp} \subseteq \operatorname{rad}\left(A^{* *}\right)$.

Summarizing, $A^{* *}$ is a Banach algebra such that $\operatorname{rad}\left(A^{* *}\right)^{2}=0$ and $A^{* *} /$ $\operatorname{rad}\left(A^{* *}\right)$ is isometrically isomorphic to $C(\Omega)$ for an extremely disconnected compact Hausdorff space $\Omega$. However $A^{* *}$ need not be commutative.

Definition 2.2. Let $\mathbb{B}_{b}(K)$ be the algebra of bounded Borel functions on $K$ and let $\Psi: \mathbb{B}_{b}(K) \rightarrow C(K)^{* *}$ be defined by

$$
\Psi(f)(\mu)=\int_{K} f d \mu \quad\left(\mu \in C(K)^{*}\right)
$$

It is easily seen that $\Psi$ is an isometric algebra monomorphism extending the natural embedding $C(K) \rightarrow C(K)^{* *}$. We shall say that an idempotent $p \in A^{* *}$ corresponds to a Borel subset $E$ of $K$ if $\pi^{* *}(p)=\Psi\left(\chi_{E}\right)$, where $\chi_{E}$ is the characteristic function for $E$.

Lemma 2.3. Suppose that $\left\{a_{\lambda}\right\}$ is a bounded net in $A$. Set $f_{\lambda}=\pi\left(a_{\lambda}\right) \in C(K)$ and suppose that the functions $f_{\lambda}$ are real and $f_{\lambda} \nearrow f$ (pointwise, increasing). Then $f$ is a Borel function and $\pi^{* *}(a)=\Psi(f)$ for every weak*-cluster point $a \in A^{* *}$ of $\left\{a_{\lambda}\right\}$.

Proof. Since the functions $f_{\lambda}$ are all continuous and bounded we have that $f \in$ $\mathbb{B}_{b}(K)$ and $\int_{K} f_{\lambda} d \mu \nearrow \int_{K} f d \mu$ for every positive measure $\mu \in M(K)$. For an arbitrary measure $\nu \in M(K)$ we obtain $\int_{K} f_{\lambda} d \nu \rightarrow \int_{K} f d \nu$ by decomposing $\nu$ into a complex linear combination of positive measures. Hence $\Psi\left(f_{\lambda}\right)$ converges weak $^{*}$ to $\Psi(f)$ in $C(K)^{* *}$.

Let $a \in A^{* *}$ be a weak*-cluster point of $\left\{a_{\lambda}\right\}$ and let $\left\{a_{\alpha}\right\}$ be a subnet converging weak * to $a$. Since $\Psi\left(f_{\alpha}\right)=\Psi\left(\pi\left(a_{\alpha}\right)\right)=\pi^{* *}\left(a_{\alpha}\right)$ we conclude that $\Psi\left(f_{\alpha}\right)$ converges weak * to $\pi^{* *}(a)$.

Lemma 2.4. Let $X$ be a Banach space, $Y \subset X$ be a closed subspace, and $q: X \rightarrow X / Y$ be the quotient map. For every $f \in(X / Y)^{* *}$ there exists $x \in X^{* *}$ such that $q^{* *}(x)=f$ and $\|x\|_{X^{* *}}=\|f\|_{(X / Y)^{* *}}$. 
Proof. First note that $(X / Y)^{* *}$ is isometrically isomorphic to $X^{* *} / Y^{\perp \perp}$. Let $x_{0} \in X^{* *}$ be any element with $q^{* *}\left(x_{0}\right)=f$. Then we have

$$
\|f\|_{(X / Y) * *}=\inf _{u \in Y^{\perp \perp}}\left\|x_{0}+u\right\|_{X^{*}}
$$

Hence there is a bounded net $\left\{u_{\lambda}\right\}$ in $Y^{\perp \perp}$ for which

$$
\|f\|_{(X / Y)^{* *}}=\lim _{\lambda}\left\|x_{0}+u_{\lambda}\right\| \text {. }
$$

By compactness we may find a weak ${ }^{*}$-converging subnet $\left\{u_{\alpha}\right\}$, say $u_{\alpha} \rightarrow u_{0} \in$ $Y^{\perp \perp}$. Now we have

$$
\|f\|_{(X / Y)^{*}} \leq\left\|x_{0}+u_{0}\right\| \leq \lim \inf _{\alpha}\left\|x_{0}+u_{\alpha}\right\|=\|f\|_{(X / Y)^{*}}
$$

\section{REDUCTION to $A \subset A^{* *}$}

We continue to let $A$ be a commutative Banach algebra such that $\operatorname{rad}(A)^{2}=0$ and $A / \operatorname{rad}(A)$ is isometrically isomorphic to $C(K)$. In this section we shall furthermore let $B$ be a closed, commutative subalgebra of $A^{* *}$ such that $A \subseteq$ $B \subseteq A^{* *}$. The aim of this section is to prove that if $B$ has (SWD), then $A$ has (SWD).

We begin by considering the radical of (not necessarily closed) subalgebras of $A^{* *}$.

Lemma 3.1. Let $\mathscr{U}$ be a unital subalgebra of $A^{* *}$. Then

$$
\operatorname{rad}(\mathscr{U})=\operatorname{rad}\left(A^{* *}\right) \cap \mathscr{U} .
$$

Proof. Clearly $\operatorname{rad}\left(A^{* *}\right) \cap \mathscr{U}$ is an ideal in $\mathscr{U}$. By Lemma 2.1 this ideal consists of nilpotent elements. Hence $\operatorname{rad}\left(A^{* *}\right) \cap \mathscr{U} \subseteq \operatorname{rad}(\mathscr{U})$.

Conversely let $u \in \operatorname{rad}(\mathscr{U})$ be arbitrary. The spectral radius of $u$ satisfies $\nu(u)=0$. Hence $\nu\left(\pi^{* *}(u)\right)=0$, and so $\pi^{* *}(u)=0$ by commutativity. We conclude that $u \in \operatorname{rad}\left(A^{* *}\right) \cap \mathscr{U}$.

Clearly it follows from the lemma that if $\mathscr{U}_{1}$ and $\mathscr{U}_{2}$ are two subalgebras of $A^{* *}$ such that $\mathscr{U}_{1} \subseteq \mathscr{U}_{2}$, then $\operatorname{rad}\left(\mathscr{U}_{1}\right)=\operatorname{rad}\left(\mathscr{U}_{2}\right) \cap \mathscr{U}_{1}$. Let $A_{0}$ be defined by $A_{0}=A+\operatorname{rad}(B)$.

Lemma 3.2. The space $A_{0}$ is a commutative subalgebra of $A^{* *}$. Moreover if $B$ has $(S W D)$, then $A_{0}$ has $(W D)$.

Proof. Since $B$ is commutative and $A \subseteq B$, we have that $\operatorname{rad}(B)$ is a symmetric $A$-bimodule. Hence $A_{0}$ is a commutative subalgebra of $A^{* *}$. Obviously we have $A_{0} \subseteq B$, and so by Lemma 3.1 we obtain

$$
\operatorname{rad}\left(A_{0}\right)=\operatorname{rad}(B) \cap A_{0}=\operatorname{rad}(B) .
$$

Suppose that $B$ has (SWD), say $B=\mathscr{U} \oplus \operatorname{rad}(B)$ for a closed subalgebra $\mathscr{U}$ of $B$, and let $a \in A_{0}$ be arbitrary. Then $a \in B$ and hence there exist unique $u \in \mathscr{U}$ and $r \in \operatorname{rad}(B)$ such that $a=u+r$. Since $\operatorname{rad}(B) \subset A_{0}$, we have $u=a-r \in A_{0} \cap \mathscr{U}$. It follows that $A_{0}$ has the Wedderburn decomposition $A_{0}=\mathscr{U}_{0} \oplus \operatorname{rad}\left(A_{0}\right)$, where $\mathscr{U}_{0}=\mathscr{U} \cap A_{0}$.

Lemma 3.3. Suppose that $X$ is a subspace of $A$ complementary to $\operatorname{rad}(A)$. Then $X$ is a subspace of $A_{0}$ complementary to $\operatorname{rad}\left(A_{0}\right)$.

Proof. It follows from Lemma 3.1 that $X \cap \operatorname{rad}\left(A_{0}\right)=(X \cap A) \cap \operatorname{rad}\left(A_{0}\right)=$ $X \cap \operatorname{rad}(A)=0$. Furthermore for $a_{0} \in A_{0}$ there exist $a \in A$ and $r \in \operatorname{rad}(B)=$ 
$\operatorname{rad}\left(A_{0}\right)$ such that $a_{0}=a+r$. Since there exist unique $x \in X$ and $r_{0} \in \operatorname{rad}(A)$ such that $a=x+r_{0}$, we obtain $a_{0}=a+r=\left(x+r_{0}\right)+r=x+\left(r_{0}+r\right)$. But $r+r_{0} \in \operatorname{rad}\left(A_{0}\right)$ so we conclude that $A_{0}=X \oplus \operatorname{rad}\left(A_{0}\right)$.

Lemma 3.4. $\operatorname{rad}\left(A_{0}\right)$ is a symmetric Banach-C(K)-bimodule and $\operatorname{rad}(A) \subseteq$ $\operatorname{rad}\left(A_{0}\right)$ is a Banach- $C(K)$-submodule.

Proof. Clearly $\operatorname{rad}(A)$ and $\operatorname{rad}\left(A_{0}\right)=\operatorname{rad}(B)$ are Banach spaces. From the fact that

$$
A_{0} / \operatorname{rad}\left(A_{0}\right)=C(K)=A / \operatorname{rad}(A)
$$

and $\operatorname{rad}\left(A_{0}\right)^{2}=0=\operatorname{rad}(A)^{2}$, it follows that $\operatorname{rad}\left(A_{0}\right)$ is a symmetric $C(K)$ bimodule for the operation $f \cdot r=r \cdot f=a_{0} r$, where $f \in C(K), r \in \operatorname{rad}\left(A_{0}\right)$ and $a_{0}$ is any element in $A_{0}$ with $\pi^{* *}\left(a_{0}\right)=f$. Clearly for this module operation $\operatorname{rad}(A)$ is a submodule. It is elementary to verify that the module operation is a Banach module operation.

The following proposition is the main result of this section.

Proposition 3.5. Suppose that there exists a closed commutative subalgebra $B$ of $A^{* *}$ such that $A \subseteq B \subseteq A^{* *}$, and such that $B$ has a strong Wedderburn decomposition. Then $A$ has (SWD).

Proof. Let $X$ be a subspace of $A$ complementary to $\operatorname{rad}(A)$, and define a map $T: C(K) \times C(K) \rightarrow \operatorname{rad}(A)$ by

$$
T(f, g)=\eta(f) \eta(g)-\eta(f g),
$$

where $\eta$ is the inverse of the map $\pi_{\mid X}: X \rightarrow C(K)$. Then $A$ is algebraically isomorphic to the algebra $C(K) \oplus \operatorname{rad}(A)$ with multiplication given by

$$
(f, r)(g, s)=(f g, T(f, g)+f \cdot s+r \cdot g),
$$

for $f, g \in C(K)$ and $r, s \in \operatorname{rad}(A)$.

Set $A_{0}=A+\operatorname{rad}(B)$. Then it follows from Lemma 3.3 that $A_{0}$ is algebraically isomorphic to the algebra $C(K) \oplus \operatorname{rad}\left(A_{0}\right)$ with multiplication given by $(f, r)(g, s)=(f g, T(f, g)+f \cdot s+r \cdot g)$, where $f, g \in C(K)$ and $r, s \in \operatorname{rad}\left(A_{0}\right)$.

From Lemma $3.2, A_{0}$ has a Wedderburn decomposition, and so by [8, Proposition 6.2] there exists a linear map $S: C(K) \rightarrow \operatorname{rad}\left(A_{0}\right)$ such that

$$
T(f, g)=f \cdot S(g)-S(f g)+S(f) \cdot g \quad(f, g \in C(K)) .
$$

Consider the quotient map $Q: \operatorname{rad}\left(A_{0}\right) \rightarrow \operatorname{rad}\left(A_{0}\right) / \operatorname{rad}(A)$, and the map $D=$ $Q \circ S$. By Lemma 3.4, $D: C(K) \rightarrow \operatorname{rad}\left(A_{0}\right) / \operatorname{rad}(A)$ is a derivation from $C(K)$ into a symmetric Banach $C(K)$-bimodule, and so $D=0$. Hence we have $S(C(K)) \subseteq \operatorname{rad}(A)$. It is easy to see that $\mathscr{U}=\{(f,-S(f)) \in C(K) \oplus \operatorname{rad}(A) \mid f \in$ $C(K)\}$ is a subalgebra of $A$ complementary to $\operatorname{rad}(A)$. By [2, Theorem 2.2], $A$ has a (SWD).

\section{Boolean ALgebras}

Let $B(K)$ be the commutative Banach algebra of all bounded complex-valued functions on $K$, and let $\mathscr{C}$ be a Boolean algebra of subsets of $K$. (See [5, Definition III.1.3].) It is elementary to check that

$$
B_{0}(K, \mathscr{C}):=\operatorname{span}\left\{\chi_{E} \mid E \in \mathscr{C}\right\}
$$


is a commutative subalgebra of $B(K)$, and that $\left\{\chi_{E} \mid E \in \mathscr{C}\right\}$ is the set of idempotents of $B_{0}(K, \mathscr{C})$.

We let $B(K, \mathscr{C})$ denote the uniform closure of $B_{0}(K, \mathscr{C})$ in $B(K)$. By [5, IV.9.10], $B(K, \mathscr{C})$ is isometrically isomorphic to $C\left(K_{1}\right)$ for a totally disconnected compact Hausdorff space $K_{1}$.

Lemma 4.1. Let $K_{1}$ be a totally disconnected, compact Hausdorff space, and let $\mathscr{E}$ be the linear span of the idempotents of $C\left(K_{1}\right)$. Suppose that $\mathscr{A} \subset C\left(K_{1}\right)$ is a subalgebra which is a commutative Banach algebra for some norm \|\|$\cdot \|$. Suppose further that $(\mathscr{A},|\|\cdot \mid\|)$ contains $\mathscr{E}$ as a dense subalgebra. Then the maximal ideal space of $\mathscr{A}$ is homeomorphic to $K_{1}$.

Proof. First note that every $x \in K_{1}$ defines a character $\phi_{x}$ on $\mathscr{A}$ by $f \mapsto$ $\phi_{x}(f)=f(x)$. The map $\tau: K_{1} \rightarrow \Phi_{\mathscr{A}}$ given by $\tau(x)=\phi_{x}$ is clearly continuous. Since $K_{1}$ is totally disconnected, $\mathscr{E}$ separates the points of $K_{1}$ and so $\tau$ is injective.

Now let $\phi \in \Phi_{\mathscr{A}}$ be arbitrary. Then $\phi$ is a non-trivial character on $\mathscr{E}$. The kernel $\Phi$ is a maximal ideal in $\mathscr{E}$. Since $\mathscr{E}$ is a selfadjoint algebra, standard arguments show that there exists $x \in K_{1}$ such that $\phi=\phi_{x}$ on $\mathscr{E}$. Since $\mathscr{E} \subset \mathscr{A}$ is dense for the norm $\|\cdot\| \|$ we have $\phi=\phi_{x}$ on $\mathscr{A}$. Hence $\tau$ is bijective, and then $\tau$ is a homeomorphism by the compactness of $K_{1}$.

Proposition 4.2. Let $\mathscr{C}$ be a Boolean algebra of Borel subsets of $K$. Suppose that there is a map $E \mapsto p_{E}, \mathscr{C} \rightarrow \mathscr{I}\left(A^{* *}\right)$ such that $\pi^{* *}\left(p_{E}\right)=\chi_{E}(E \in \mathscr{C})$, and suppose that $p_{E} p_{F}=p_{F} p_{E}=0$ whenever $E \cap F=\varnothing$. Set $B_{0}=\operatorname{span}\left\{p_{E} \mid E \in \mathscr{C}\right\}$ and $B=\bar{B}_{0}$. Then we have

(i) $B_{0}$ is a commutative algebra isomorphic to $B_{0}(K, \mathscr{C})$;

(ii) $B$ is a commutative Banach algebra such that $\Phi_{B}$ is totally disconnected and $\mathscr{I}(B)=\left\{p_{E} \mid E \in \mathscr{C}\right\}$.

Proof. (i) Let 1 be the identity in $A^{* *}$. First note that, if $F \in \mathscr{C}$ and $E=$ $K \backslash F$, then $q_{1}=p_{E}+p_{F} \in \mathscr{U}$ is an idempotent such that $\pi\left(q_{1}\right)=\chi_{K}=1_{C(K) * *}$. Hence $q_{1}=1$, and so $p_{E}=1-p_{F}$. Next let $E, F \in \mathscr{C}$ be disjoint. Then $q_{2}=p_{E}+p_{F}$ is an idempotent such that $\pi\left(q_{2}\right)=\chi_{E \cup F}$. Obviously we have $q_{2} p_{K \backslash(E \cup F)}=p_{K \backslash(E \cup F)} q_{2}=0$. Hence as before $q_{2}=1-p_{K \backslash(E \cup F)}=p_{E \cup F}$.

Now let $E, F \in \mathscr{C}$ be arbitrary. From what we just proved it follows that

$$
p_{E}=p_{E \cap F}+p_{E \backslash(E \cap F)} \text {. }
$$

We also have $\left(1-p_{F}\right) p_{E \cap F}=p_{K \backslash F} p_{E \cap F}=0$. Hence

$$
p_{F} p_{E \cap F}=p_{E \cap F} \text {. }
$$

Finally from (1) and (2) we obtain $p_{F} p_{E}=p_{E \cap F}=p_{E} p_{F}$. Now it is not difficult to see that every element $a$ in $B_{0}$ has the form

$$
a=\sum_{j=1}^{n} \lambda_{j} p_{E_{j}},
$$

where $\lambda_{1}, \ldots, \lambda_{n} \in \mathbb{C}$ and $E_{1}, \ldots, E_{n} \in \mathscr{C}$ form a partition of $K$. From this (i) follows.

(ii) From Lemma 3.1 it follows that $B / \operatorname{rad}(B)$ is isomorphic to $\pi^{* *}(B)$. Obviously we have

$$
B_{0}(K, \mathscr{C})=\pi^{* *}\left(B_{0}\right) \subseteq \pi^{* *}(B) \subseteq B(K, \mathscr{C}) .
$$


From this and Lemma 4.1 it follows that $\pi^{* *}(B)$ has totally disconnected maximal ideal space, so the same is true for $B$. Since $B$ is commutative, $\pi^{* *}$ establishes a one-one correspondence between the idempotents in $B$ and the idempotents of $B(K, \mathscr{C})$. Hence we have $\mathscr{I}(B)=\left\{p_{E} \mid E \in \mathscr{C}\right\}$.

Theorem 4.3. Let $A$ be a unital commutative Banach algebra with $\operatorname{rad}(A)^{2}=0$. Suppose that $A / \operatorname{rad}(A)$ is isomorphic to $C(K)$ for some compact Hausdorff space $K$. Suppose that the following conditions hold:

(i) There is a Boolean algebra $\mathscr{C}$ of Borel subsets of $K$ with $C(K) \subseteq$ $B(K, \mathscr{C})$.

(ii) There is a bounded set of idempotents $\mathscr{I}=\left\{p_{e} \mid E \in \mathscr{C}\right\}$ in $A^{* *}$ such that $\pi^{* *}\left(p_{E}\right)=\chi_{E}$ for all $E \in \mathscr{C}$, and $p_{E} p_{F}=0$ whenever $E \cap F=\varnothing$.

Then $A$ has a strong Wedderburn decomposition.

Proof. Set $B_{0}=\operatorname{span}(\mathscr{I})$ and $B=\bar{B}_{0}$. We have that $B$ is a commutative Banach algebra such that $\mathscr{I}(B)$ is bounded and $\Phi_{B}$ is totally disconnected. By [1, Theorem 4.2] it follows that $B$ has (SWD) and $B / \operatorname{rad}(B)$ is isomorphic to $C\left(\Phi_{B}\right)$. Furthermore $B$ is the closed linear span of its idempotents, hence by [3, Lemma 3.3] $B$ is semisimple. Consequently $\pi^{* *}: B \rightarrow B(K, \mathscr{C})$ is an isomorphism.

Now define $\mathfrak{A}$ to be the closed subalgebra of $A^{* *}$ generated by $A$ and $B$. Since $A$ is in the centre of $A^{* *}$, it follows that $\mathfrak{A}$ is a commutative, closed subalgebra of $A^{* *}$ containing $A$. We have that

$$
\mathfrak{A} / \operatorname{rad}(\mathfrak{A})=\mathfrak{A} /\left(\mathfrak{A} \cap \operatorname{rad}\left(A^{* *}\right)\right)
$$

and the latter space is isomorphic to $\pi^{* *}(\mathfrak{A})$.

Since $B \subseteq \mathfrak{A}$ we have

$$
B(K, \mathscr{C})=\pi^{* *}(B) \subseteq \pi^{* *}(\mathfrak{A}) .
$$

On the other hand let $\mathfrak{A}_{0}$ be the (non-closed) algebra generated in $A^{* *}$ by $A$ and $B$. Then obviously $\overline{\mathfrak{A}_{0}}=\mathfrak{A}$. For each element $u$ of $\mathfrak{A}_{0}$ there are elements $a_{1}, \ldots, a_{n} \in A$ and $b_{1}, \ldots, b_{n} \in B$ such that

$$
u=\sum_{j=1}^{n} a_{n} b_{n}
$$

Hence $\pi^{* *}(u)=\sum_{j=1}^{n} \pi^{* *}\left(a_{n}\right) \pi^{* *}\left(b_{n}\right) \in B(K, \mathscr{C})$, because $\pi^{* *}(A)=C(K) \subset$ $B(K, \mathscr{C})$. We conclude that $\pi^{* *}\left(\mathfrak{A}_{0}\right) \subset B(K, \mathscr{C})$, and so

$$
\pi^{* *}(\mathfrak{A})=B(K, \mathscr{C}) .
$$

Since $\operatorname{rad}(\mathfrak{A})^{2}=0$ we have by [1, Theorem 4.2] that $\mathfrak{A}$ has (SWD). The result now follows from Proposition 3.5.

\section{SOME LEMMAS ON IDEMPOTENTS IN $A^{* *}$}

Lemma 5.1. For every open set $G \subseteq K$ there exists $a_{G} \in A^{* *}$ with $\left\|a_{G}\right\| \leq 1$ and $\pi^{* *}\left(a_{G}\right)=\chi_{G}$. Furthermore if $G, H \subseteq K$ are disjoint open sets and $b \in A^{* *}$, then

$$
a_{G} b a_{H}=0 .
$$

Proof. Let $G$ be a given open set and let $\left\{f_{\lambda}\right\}$ be a net of positive functions in $C(K)$ with $\operatorname{supp}\left(f_{\lambda}\right) \subseteq G$ and $f_{\lambda} \nearrow \chi_{G}$, and choose a bounded net of positive 
reals $\left\{t_{\lambda}\right\}$ such that $t_{\lambda} \rightarrow 0$. For each $\lambda$ we have $\left\|f_{\lambda}\right\|_{\infty} \leq 1$, and so we may choose $a_{\lambda}(G) \in A$ with $\pi\left(a_{\lambda}(G)\right)=f_{\lambda}$ and $\left\|a_{\lambda}(G)^{2}\right\| \leq\left\|a_{\lambda}(G)\right\|^{2} \leq 1+t_{\lambda}$. Let $a_{G} \in A^{* *}$ be a weak*-cluster point of $\left\{a_{\lambda}(G)^{2}\right\}$. It follows that $\left\|a_{G}\right\| \leq 1$, and by Lemma 2.3 we have $\pi^{* *}\left(a_{G}\right)=\chi_{G}$.

Let $G$ and $H$ be disjoint open sets and let $b \in A^{* *}$ be arbitrary. Let $\left\{a_{\lambda}(G)\right\}$ and $\left\{a_{\rho}(H)\right\}$ be the nets just specified and let $\left\{b_{\alpha}\right\}$ be any net in $A$ converging weak * to $b$. Then we have

$$
\begin{aligned}
a_{G} b a_{H} & =\lim \lim _{\lambda^{\prime}} \lim _{\rho^{\prime}} a_{\lambda^{\prime}}(G)^{2} b_{\alpha} a_{\rho^{\prime}}(H)^{2} \\
& =\lim _{\lambda^{\prime}} \lim _{\alpha} \lim _{\rho^{\prime}} b_{\alpha}\left(a_{\lambda^{\prime}}(G) a_{\rho^{\prime}}(H)\right)^{2}
\end{aligned}
$$

for suitable subnets $\left\{a_{\lambda^{\prime}}(G)\right\}$ and $\left\{a_{\rho^{\prime}}(H)\right\}$.

Since $\operatorname{supp}\left(\pi^{* *}\left(a_{\lambda}(G)\right)\right) \subseteq G$ and $\operatorname{supp}\left(\pi^{* *}\left(a_{\rho}(H)\right)\right) \subseteq H$ we have

$$
a_{\lambda}(G) a_{\rho}(H) \in \operatorname{rad}\left(A^{* *}\right),
$$

and hence

$$
a_{G} b a_{H}=0
$$

because $\operatorname{rad}\left(A^{* *}\right)^{2}=0$.

Note that it follows from the lemma that $a_{G} a_{H}=0$ when $G$ and $H$ are disjoint open sets.

Lemma 5.2. Let $p, q \in A^{* *}$ be idempotents corresponding to Borel subsets of $K$ with disjoint closures. Then $p q=q p=0$.

Proof. Set $a=(1-q) p(1-q)$. Then $\pi^{* *}(a)=\pi^{* *}(p)$ and $a q=q a=0$. Set $p_{0}=3 a^{2}-2 a^{3}$. Then $p_{0}$ is an idempotent with $p_{0} q=q p_{0}=0$ and $p=p_{0}+r$ for some $r \in \operatorname{rad}\left(A^{* *}\right)$. Hence

$$
p=p^{2}=p_{0}+p_{0} r+r p_{0} .
$$

It follows that $p q=p_{0} r q=b r q$ for all $b \in A^{* *}$ with $\pi^{* *}(b)=\pi^{* *}(p)$ since $\operatorname{rad}\left(A^{* *}\right)^{2}=0$.

By assumption $\pi^{* *}(p)=\chi_{E}$ and $\pi^{* *}(q)=\chi_{F}$ for subsets $E, F$ of $K$ such that $\bar{E} \cap \bar{F}=\varnothing$. From normality of $K$ we may find $f \in C(K)$ such that $f(\bar{E})=1$ and $f(\bar{F})=0$. Choose $c \in A$ with $\pi(c)=f$. Then we have $\pi^{* *}(p c)=\pi^{* *}(p) f=\pi^{* *}(p)$ and $\pi^{* *}(c q)=f \chi_{F}=0$. Hence $c q \in \operatorname{rad}\left(A^{* *}\right)$. Set $b=p c$. Then

$$
p q=b r q=p c r q=p r c q=0,
$$

since $A$ is in the centre of $A^{* *}$ and $\operatorname{rad}\left(A^{* *}\right)^{2}=0$. The fact that $q p=0$ follows by symmetry.

Lemma 5.3. For every idempotent $e \in A^{* *} / \operatorname{rad}\left(A^{* *}\right)$ there exists an idempotent $p \in A^{* *}$ such that $\pi^{* *}(p)=e$ and $\|p\|=1$.

Proof. By Lemma 2.4 there exists $a \in A^{* *}$ with $\pi^{* *}(a)=e$ and $\|a\|=\|e\|=1$. Then $\pi^{* *}\left(a^{n+2}\right)=\pi^{* *}(a)^{n+2}=e$, and so $1 \leq\left\|a^{n+2}\right\| \leq\|a\|^{n+2} \leq 1$.

Let $p_{0}$ be any idempotent in $A^{* *}$ such that $\pi^{* *}\left(p_{0}\right)=e$, and choose $r \in$ $\operatorname{rad}\left(A^{* *}\right)$ such that $a=p_{0}+r$. By induction note that for all $n \in \mathbb{N} \cup\{0\}$ we have

$$
a^{n+2}=\left(p_{0}+r\right)^{n+2}=p_{0}+p_{0} r+r p_{0}+n p_{0} r p_{0} .
$$


Set $u=p_{0}+p_{0} r+r p_{0}$ and $v=p_{0} r p_{0}$. Then $u=a^{2}$ and so $\|u\|=1$. For every $n \in \mathbb{N}$ we have $\|u+n v\|=1$ and then $n\|v\|=\|n v+u-u\| \leq\|n v+u\|+\|u\|=2$. Hence $p_{0} r p_{0}=v=0$. Consequently $p=a^{2}=\left(p_{0}+r\right)^{2}=p_{0}+p_{0} r+r p_{0}$ is an idempotent of norm 1 with $\pi^{* *}(p)=e$.

\section{THE CASE $K=[0,1]$}

In this section we restrict ourselves to the case where $K=[0,1]$. That is, $A$ is a commutative Banach algebra with $R^{2}=0$ and $A / R$ isometrically isomorphic to $C([0,1])$.

For $x \in K$ let $p_{x} \in A^{* *}$ be an idempotent of norm 1 corresponding to $K \backslash\{x\}$, and set $q_{x}=1-p_{x}$. Then for each $x \in K, q_{x}$ is an idempotent corresponding to the set $\{x\}$. Hence from Lemma 5.2 it follows that $\left\{q_{x} \mid x \in\right.$ $K\}$ is an orthogonal set of idempotents.

Let $x, y \in K$ be arbitrary with $x \neq y$. Then we have

$$
1-p_{x}-p_{y}+p_{x} p_{y}=q_{x} q_{y}=0=q_{y} q_{x}=1-p_{x}-p_{y}+p_{y} p_{x},
$$

and so $p_{x}, p_{y}$ commute.

Proposition 6.1. Let $\mathscr{F}$ be the family of open subsets $K$ with finite boundary. Then there is a map $G \mapsto Q_{G}$ from $\mathscr{F}$ into $\mathscr{I}\left(A^{* *}\right)$ satisfying.

(i) $\pi^{* *}\left(Q_{G}\right)=\chi_{G}$,

(ii) $\left\|Q_{G}\right\| \leq 5$,

(iii) $Q_{G} Q_{H}=0$ whenever $G$ and $H$ are disjoint,

(iv) $q_{x} Q_{G}=Q_{G} q_{x}=0$ for all $x \in K \backslash G$.

Proof. Let $G \in \mathscr{F}$ be arbitrary, and let $a_{G}$ be as in Lemma 5.1. Since $\left\{p_{x} \mid x \in\right.$ $K\}$ is a commuting set of idempotents we may define an element $b_{G} \in A^{* *}$ by

$$
b_{G}=\left(\prod_{x \in \partial(G)} p_{x}\right) a_{G}\left(\prod_{x \in \partial(G)} p_{x}\right) \text {. }
$$

Note that

$$
\begin{aligned}
\pi^{* *}\left(b_{G}\right) & =\left(\prod_{x \in \partial(G)} \pi^{* *}\left(p_{x}\right)\right) \pi^{* *}\left(a_{G}\right)\left(\prod_{x \in \partial(G)} \pi^{* *}\left(p_{x}\right)\right) \\
& =\left(\prod_{x \in \partial(G)}\left(1-\chi_{\{x\}}\right)\right) \chi_{G}\left(\prod_{x \in \partial(G)}\left(1-\chi_{\{x\}}\right)\right) \\
& =\chi_{G} .
\end{aligned}
$$

An easy calculation shows that $Q_{G}=3 b_{G}^{2}-2 b_{G}^{3}$ is an idempotent such that $\pi^{* *}\left(Q_{G}\right)=\chi_{G}$. Clearly we have $\left\|b_{G}\right\| \leq 1$, and so $\left\|Q_{G}\right\| \leq 3\left\|b_{G}\right\|^{2}+2\left\|b_{G}\right\|^{3} \leq 5$.

Let $G, H \subset K$ be disjoint open sets with finite boundaries. Then it is easily seen from Lemma 5.1 that $Q_{G} Q_{H}=Q_{H} Q_{G}=0$.

Let $G \subset K$ be an open set with finite boundary and let $x \in K \backslash \bar{G}$. Then (iv) follows from Lemma 5.2. On the other hand if $x \in \partial(G)$, then we have

$$
q_{x} Q_{G}=q_{x} p_{x} Q_{G}=0=Q_{G} p_{x} q_{x}=Q_{G} q_{x}
$$

Observe that it is important that $\left\|p_{x}\right\|=1$. Otherwise the norm of $b_{G}$ could "blow up". This is where we make use of the fact that $A / R$ is not 
only isomorphic to $C(K)$ but is assumed to be isometrically isomorphic to $\left(C(K),\|\cdot\|_{\infty}\right)$.

Lemma 6.2. Let $\left\{x_{1}, \ldots, x_{n}\right\}$ be a finite set of (distinct) points in $K$. Then $\left\|\sum_{j=1}^{n} q_{x_{j}}\right\| \leq 6$.

Proof. Set $q=\sum_{j=1}^{n} q_{x_{j}}$. Then $q$ is an idempotent such that $\pi^{* *}(q)=$ $\chi_{\left\{x_{1}, \ldots, x_{n}\right\}}$. If $G=K \backslash\left\{x_{1}, \ldots, x_{n}\right\}$, then $G$ is an open set with finite boundary. From the fact that $q$ and $Q_{G}$ are orthogonal, we see that $q=1-Q_{G}$. Hence $\left\|\sum_{j=1}^{n} q_{x_{j}}\right\| \leq 6$.

For a subset $S \subseteq K$ let $S^{\circ}$ denote the interior of $S$. Note that $\partial\left(S^{\circ}\right) \subseteq$ $\partial(S)$. Hence if $S \subseteq K$ has finite boundary, then $S^{\circ}$ is an open set with finite boundary.

We now prove our main result.

Theorem 6.3. Let $A$ be a unital commutative Banach algebra with $\operatorname{rad}(A)^{2}=0$. Suppose $A / \operatorname{rad}(A)=\left(C([0,1]),\|\cdot\|_{\infty}\right)$. Then $A$ has $(S W D)$.

Proof. Let $K=[0,1]$, and let $\mathscr{C}$ denote the collection of sets $S \subset K$ with finite boundary. Then $\mathscr{C}$ is a Boolean algebra of Borel sets of $K$, and since $\mathscr{C}$ contains the intervals it is easily seen that $C(K) \subseteq B(K, \mathscr{C})$. (This argument is the only one where we need $K=[0,1]$.)

For every $S \in \mathscr{C}$ we define

$$
P_{S}=Q_{S^{\circ}}+\sum_{x \in(\partial(S) \cap S)} q_{x},
$$

and let $\mathscr{I}$ be the set $\left\{P_{S} \mid S \in \mathscr{C}\right\}$. Then it follows from Lemma 6.2 and Proposition 6.1 that $\mathscr{I}$ is a bounded set of idempotents such that $\pi^{* *}\left(P_{S}\right)=\chi_{S}$ for all $S \in \mathscr{C}$. Furthermore if $S_{1}, S_{2} \in \mathscr{C}$ are disjoint sets, then $P_{S_{1}} P_{S_{2}}=$ $P_{S_{2}} P_{S_{1}}=0$. Hence the result follows from Theorem 4.3.

\section{ACKNOWLEDGMENT}

The material presented here forms part of the author's Ph.D. thesis submitted to the University of Leeds. The author wishes to express his gratitude to his supervisor, Professor H. G. Dales, for guidance and encouragement, and to Professor W. G. Bade for helpful discussions.

\section{REFERENCES}

1. W. G. Bade and P. C. Curtis, Jr., The Wedderburn decomposition of commutative Banach algebras, Amer. J. Math. 82 (1960), 851-866.

2. W. G. Bade, The Wedderburn decomposition for quotient algebras arising from sets of nonsynthesis, Proc. Centre Math. Anal. Austral. Nat. Univ. 21 (1989), 25-31.

3. W. G. Bade and H. G. Dales, The Wedderburn decomposability of some commutative Banach algebras, J. Funct. Anal. 107 (1992), 105-121.

4. F. F. Bonsall and J. Duncan, Complete normed algebras, Ergeb. Math. Grenzgeb., vol. 80, Springer-Verlag, Berlin and New York, 1973.

5. N. Dunford and J. T. Schwartz, Linear operators, Part 1, Wiley Classics Library, Wiley, New York, 1988.

6. C. Feldman, The Wedderburn principal theorem in Banach algebras, Proc. Amer. Math. Soc. 2 (1951), 771-777. 
7. A. Ya. Helemskii, The homological dimension of normed modules over Banach algebras, Math. USSR-Sb. 10 (1970), 399-411.

8. G. Hochschild, On the cohomology groups of an associative algebra, Ann. of Math. 46 (1945), 58-67.

9. B. E. Johnson, Approximate diagonals and cohomology of certain annihilator Banach algebras, Amer. J. Math. 94 (1972), 685-698.

10. H. Kamowitz, Cohomology groups of commutative Banach algebras, Trans. Amer. Math. Soc. 102 (1962), 352-372.

11. M. Solovej, Wedderburn decompositions and cohomology for Banach algebras, Ph.D. thesis, University of Leeds, 1993.

12. _ The cohomology comparison problem for Banach algebras, J. London Math. Soc. (to appear).

Mathematics Institute, Universitet of Copenhagen, UNiversitetsparken 5, 2100 Kobenhave $\varnothing$, DenMaRK

E-mail address: solovej@math.ku.dk 